\title{
Surgical Management of Ectopic Third Molar in the Mandibular Sub condylar Region without a Pathological Cause: A Case Report
}

\author{
Sonia Nath ${ }^{1}$, Shaju Jacob Pulikkotil ${ }^{*}$, Jayant Prakash ${ }^{3}$ and Om Prakash ${ }^{4}$ \\ ${ }^{1}$ Department of Dentistry, Rajendra Institute of Medical Sciences, India. \\ ${ }^{2}$ Department of Restorative dentistry, International Medical University, Malaysia \\ ${ }^{3}$ Private Practice, India \\ ${ }^{4}$ Department of Oral Surgery, Dental Institute, Rajendra Institute of Medical Sciences, India
}

Received: August 15, 2017; Published: September 08, 2017

*Corresponding author: Shaju J Pulikkotil, International Medical University, School of Dentistry, Kuala Lumpur, Malaysia 57000, Tel: +603-86567228; Email: Shaju_Jacob@imu.edu.my

\begin{abstract}
Ectopic eruption of mandibular third molar in the subcondylar region is an extremely rare condition. The impacted tooth can remain asymptomatic throughout life time and intervention would only be required in cases of pain, signs of infection, inflammation or cyst formation. In our case a 35 year old man presented with dull continuous pain in left side of face near preauricular area. A radiographic examination revealed an impacted ectopic third molar in left subcondylar region with a radiolucency surrounding the crown. The tooth was surgically removed by an intraoral approach through a bone tunnel made on the buccal surface inferior to sigmoid notch. Inadvertently left subcondylar region fractured during the procedure and intermaxillary fixation was done with Erich arch bar. The treatment should be meticulously planned on the basis of the position and type of the ectopic tooth and related potential trauma against the benefits of surgical intervention.
\end{abstract}

Keywords: Ectopic Tooth Eruption; Third Molar Abnormalities; Tooth Impaction; Mandibular Condyle; Surgery

\section{Introduction}

Ectopic third molar teeth are those that are impacted in unusual positions or that have been displaced and are at a distance from their normal anatomic location [1]. It is common have to ectopic eruption of a tooth within the oral cavity but rare in other sites of the jaw [2]. Reports are present with teeth eruption in the nasal septum, ascending ramus of mandible, mandibular condyle, and lower border of mandible, coronoid process, sigmoid notch, palate and maxillary sinus [1-3]. Unilateral as well as bilateral ectopic impacted third molar teeth have been reported in various parts of the mandibular ramus [4].

Ectopic mandibular third molar are rare and are usually found because of clinical symptoms or accidently discovered on radiographs [3-6]. But in few cases it may cause acute infections, inflammation, sinus formation, and complicate to form cyst which would necessitate the removal of impacted tooth [2,4]. Till date very few cases of ectopic mandibular third molar have been described in the subcondylar area due to rarity [1,3-7]. The risk of damaging the neuronal structures and the joint components, esthetic concerns, defect reconstruction after the surgery, and the age of the patient should be evaluated before treatment. This report describes a case of ectopic eruption of mandibular third molar in subcondylar region without any pathological condition and describes a intraoral surgical technique for removal of tooth and its related complication.

\section{Case Description}

A 35 year old male patient reported to the department of oral surgery, Dental Institute, Rajendra Institute of Medical Sciences in June, 2017. He complained of pain in left side of face and mild discomfort in the mucosa since the last 2 months. On examination the patient experienced pain in the preauricular region. The pain was dull and continuous is nature, with occasional episodes of severe pain lasting for few hours. Clinical intraoral and extra oral examination showed no other signs of associated pathology like swelling, trismus, and redness, and fever, formation of a sinus or a fistula. Results of intraoral examination were unremarkable except for poor oral hygiene and missing left mandibular second molar which was extracted last year due to caries. The patient's medical 
history was not significant and there was no apparent systemic problem. The patient was neither alcohol nor tobacco user. The blood investigation was within normal limits.

A panoramic radiograph was taken showing the lower left third molar to be located in the subcondylar region. The impacted tooth was well formed and the crown was surrounded by radiolucent area. The ectopic impacted tooth was horizontal in position with its root apices positioned towards posterior border and crown towards the anterior border (Figure 1). According to the orientation and location of the impacted tooth it was considered as an ectopic impaction of mandibular third molar. Surgical procedure was performed under local anaesthesia, with inferior alveolar nerve block, lingual, long buccal and local infiltration. Intraoral access was selected using ward's extended incision giving exposure to the buccal and lingual surface of angle and ascending ramus. Coronoid process was located and exposed (Figure 2). The tooth was completely surrounded and covered with bone.

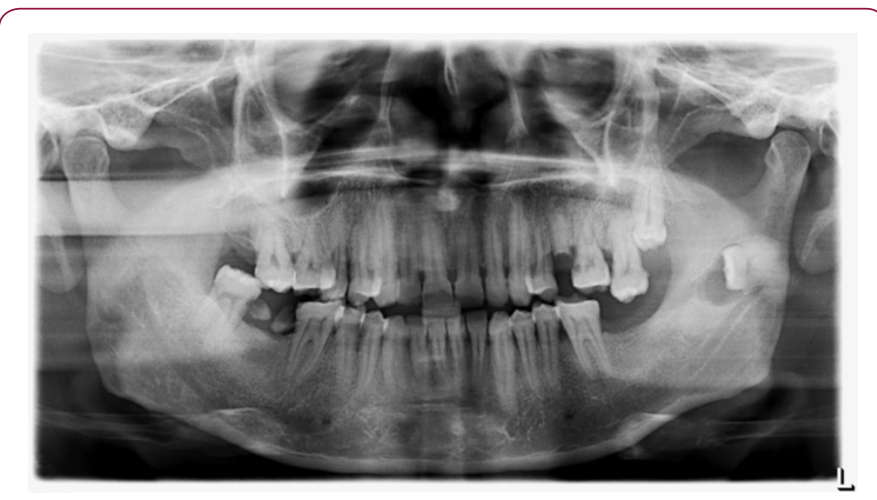

Figure 1: Panoramic radiograph showing impacted ectopic third molar in left side subcondylar region.

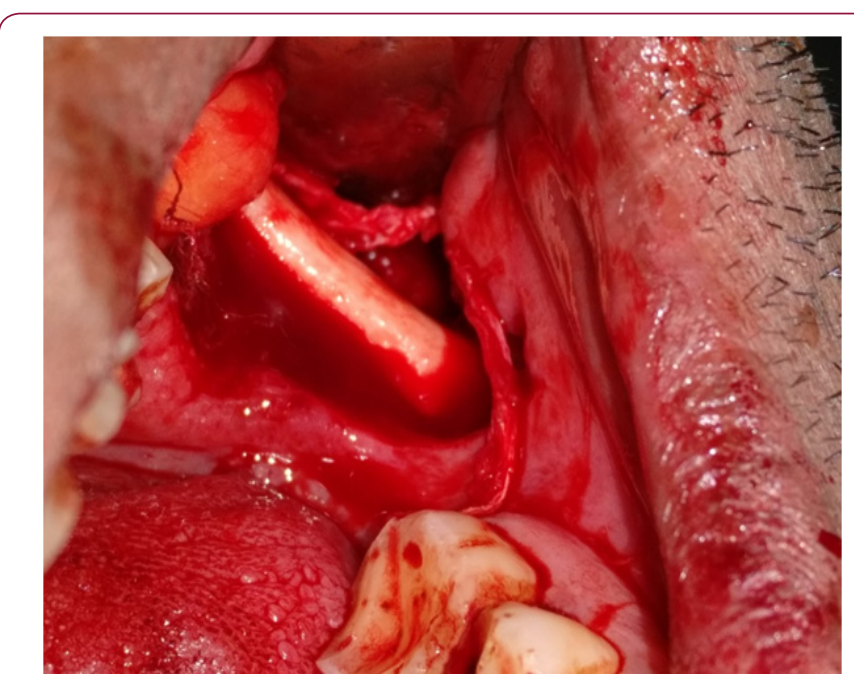

Figure 2: Coronoid process exposed with tunnel made on the buccal side.

A bony tunnel was made on the buccal surface inferior to sigmoid notch and widened with the combined use of surgical drill and chisel and mallet. The tooth was elevated carefully as the bony window was enlarged. During the procedure the mandible fractured inadvertently in the left subcondylar region. After proper debridement the wound was closed with 3'0 black silk suture. Intermaxillary fixation was done with Erich arch bar for a period of 45 days (Figure 3). Intravenous antibiotics and analgesics were prescribed for seven days. Patient was recalled after every week for 60 days. Facial swelling and ear pain on left side was noted during first week which resolved gradually. There was slight paresthesia in the post operative period, which recovered gradually after 2 months. After removal of intermaxillary fixation the patient resumed normal masticatory function after one month.

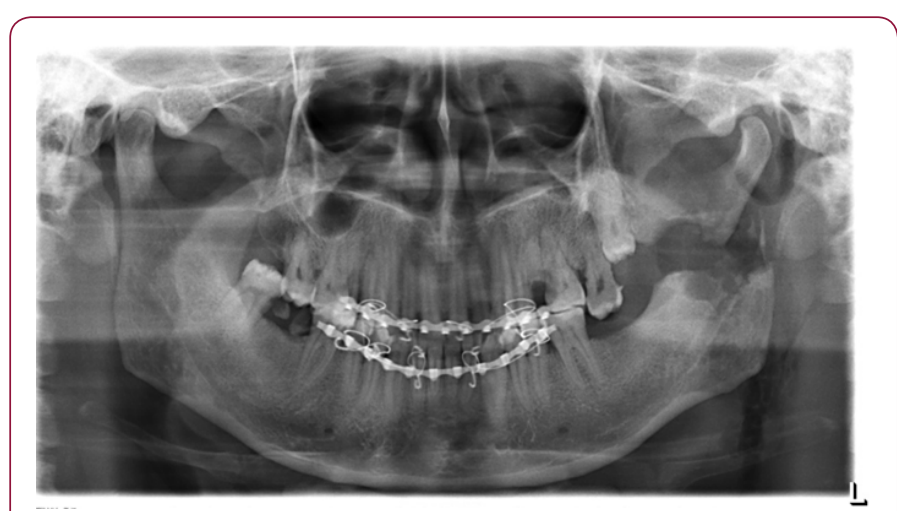

Figure 3: Post operative panoramic radiograph revealing condylar fracture on left side.

\section{Discussion}

Impaction of a tooth is a situation in which an unerupted tooth is wedged against another tooth (or teeth) or otherwise located so that it cannot erupt normally [8]. Mandibular third molar is the most frequently impacted tooth in the jaw with a frequency of $20-30 \%$ and categorized according to the anterior posterior space between the second molar and the mandibular ramus, its medial lateral position in the body of the mandible, and the position of its long axis [8]. Due to rarity of the condition the knowledge about its etiology, clinical features, therapeutic options, and surgical approaches for extraction is limited [6]. This is probably one of the few reported case of impacted third molar in the subcondylar region without any associated pathology. Inglesias-Martin et al. [6] documented 14 cases of ectopic condylar impaction, representing a sample of 15 patients from 1980-2011. In 14 patient radiolucent lesions present on radiograph were confirmed as odontogenic cyst histopathologically. In a literature review by Shivashankara et al. [7] reported 14 cases of ectopic third molar in condylar region.

In a literature review by Wang et al. [9] reported 13 cases of which 7 cases were of ectopic third molar in the subcondylar area from 1978 to 2003. Eight of the 11 cases included description of a radiolucent image around the ectopic molar on the radiograph including five with the diagnosis of dentigerous cyst. Salmeron et al. [3] reported 2 cases of third molars in the subcondylar region which were associated with odontogenic cyst. Kim [1] and Okuyama et al. [10] reported the only case of ectopic third molar in condyle without any associated pathological condition. 
The true incidence and etiology of impacted ectopic molars remain unclear. Nagarajappa and Manjunatha [2] reported the incidence to be $1 \%$ in general population. Several theories have been suggested to explain the pathogenesis. The few possibilities are;

a. Abnormal displacement of tooth bud in embryonic life, long eruption pathway,

b. Obstruction of the eruption path due to supernumerary teeth, odontomas, scar tissue, odontogenic tumor or cyst,

c. Defects in the follicle due to trauma, surgery, congenital diseases or other causes,

d. Endocrine disorder, infections, hereditary factors [3,5-9].

A three dimensional imaging is important in the management of ectopic impacted tooth as it can identify the position of the tooth, associated pathology and identify the position of neurovascular structures [1,5]. A computed tomography was not possible in our patient due to financial constraint of the patient. In most ectopic impaction, the tooth may remain asymptomatic over the course of lifetime and these patients should be followed up and monitored annually. Intervention is required in cases where the tooth is associated with severe pain, signs of acute inflammation or forming into a cyst. In our case the patient was experiencing pain and radiographically the impacted tooth was surrounded by a radiolucent area. The ectopic impacted third molar was planned for surgical removal to prevent further complications like acute inflammation, cystic lesion, diffuse osteolysis/ osteomyellitis, pathologic fracture/ deformity of condylar process or bone absorption [1,11,12]. Many investigators have reported that patients with unerupted mandibular third molars were more likely to have angle fracture than those with erupted mandibular third molar as mandibular angle is weakened by the decrease in bony space caused by presence of the tooth [13].

The choice of surgical approach depends on the preference of the oral surgeon and the location of tooth. The aim of surgical intervention should be to cause minimum morbidity without affecting the functional efficiency of the mandibular condyle. Three surgical approaches have been explained in the literature for the removal of ectopic third molar, intra or trans oral, extra oral and endoscopically assisted $[6,9,10,11,14]$. In intraoral approach the tooth is retrieved either from buccal or lingual side or sometimes through sagittal spit osteotomy procedure. Extra oral approach is usually used either submandibular or preauricular depending on impacted tooth. In endoscopically assisted techniques fiber optic technology used with endoscope for the surgical removal. Intra oral approach is the most common approach for the impacted mandibular third molar. Advantageous with this approach is aesthetically good without any extra oral scar and preserves the neurovascular bundle. However, in case of deeply impacted teeth there is increased risk of damage to inferior alveolar nerve, significant alveolar bone loss and risk of damaging the adjacent teeth. We used the intraoral approach through a tunnel made on the buccal side. Similar procedure has been used by other authors $[5,11,12]$.

Very few case reports have outlined the serious complication associated with removal of ectopic tooth. In our report the condyle was fractured during removal of impacted ectopic tooth possibly due to osteotomy procedure and the position of the tooth. Condylar region of the mandible is the most vulnerable site to fracture as this area is the weakest against the impact acting on the mandible. Intermaxillary fixation was done with the use of Erich arch bar. Similarly Pace et al. [14] used miniplates to avoid fractures postoperatively due to loss of bone support at the condylar neck post surgery.

Ectopic third molar in the subcondylar region without any associated pathology is very rare. Treatment of ectopic third molars in the coronoid and condylar regions is recommended to avoid the morbidity caused by infection of the cyst, malfunction of the temporomandibular joint, and risk of fracture in an area with a very thin bone. The decision for removal of the impacted tooth should be meticulously planned understanding the potential risk, possible complication and the benefits of the procedure. The surgical approach must be carefully planned for the aim of choosing the most conservative technique that produces the minimum trauma to patients.

\section{References}

1. Kim JS (2011) Cone beam computed tomography findings of ectopic mandibular third molar in the mandibular condyle: report of a case. Imaging Sci Dent 41(3): 135-137.

2. Nagarajappa D, Manjunatha B (2011) Tooth in oropharynx. J Oral Maxillofac Pathol 15(3): 346-347.

3. Salmerón JI, del Amo A, Plasencia J, Pujol R, Vila CN (2008) Ectopic third molar in condylar region. Int J Oral Maxillofac Surg 37: 398-400.

4. Burton DJ, Scheffer RB (1980) Serratia infection in a patient with bilateral subcondylar impacted third molars and associated dentigerous cysts: report of case. J Oral Surg 38(2): 135-138.

5. Bortoluzzi MC, Manfro R (2010) Treatment for ectopic third molar in the subcondylar region planned with cone beam computed tomography: a case report. J Oral Maxillofac Surg 68(4): 870-872.

6. Iglesias Martin F, Infante Cossio P, Torres Carranza E, Prats Golczer VE, Garcia Perla Garcia A (2012) Ectopic third molar in the mandibular condyle: a review of the literature. Med Oral Patol Oral Cir Bucal 17(6): e1013-e1017.

7. Shivashankara C, Manjunatha BS, Tanveer A (2012) Ectopic mandibular third molar in subcondylar region: report of a rare case. Oral Maxillofac Surg 16(1): 153-155.

8. Abu El Naaj I, Braun R, Leiser Y, Peled M (2010) Surgical approach to impacted mandibular third molars operative classification. J Oral Maxillofac Surg 68(3): 628-633.

9. Wang CC, Kok SH, Hou LT, Yang PJ, Lee JJ, et al. (2008) Ectopic mandibular third molar in the ramus region: report of a case and literature review. Oral Surg Oral Med Oral Pathol Oral Radiol Endod 105: 155-161.

10. Okuyama K, Sakamoto Y, Naruse T, Kawakita A, Yanamoto S, et al. (2016) Intra oral extraction of an ectopic mandibular third molar detected in the subcondylar region without a pathological cause: A case report and literature review. Cranio 3: 1-5. 
11. Gadre KS, Waknis $P$ (2010) Intra-oral removal of ectopic third molar in the mandibular condyle. Int J Oral Maxillofac Surg 39(3): 294-296.

12. Lambade P, Lambade D, Dolas RS, Virani N (2013) Ectopic mandibular third molar leading to osteomyelitis of condyle: a case report with literature review. Oral Maxillo fac Surg 17(2): 127-130.
13. Duan DH, Zhang Y (2008) Does the presence of mandibular third molars increase the risk of angle fracture and simultaneously decrease the risk of condylar fracture? Int J Oral Maxillofac Surg 37(1): 25-28.

14. Pace C, Holt D, Payne M (2010) An unusual presentation of an ectopic third molar in the condylar region. Aust Dent J 55(3): 325-327.

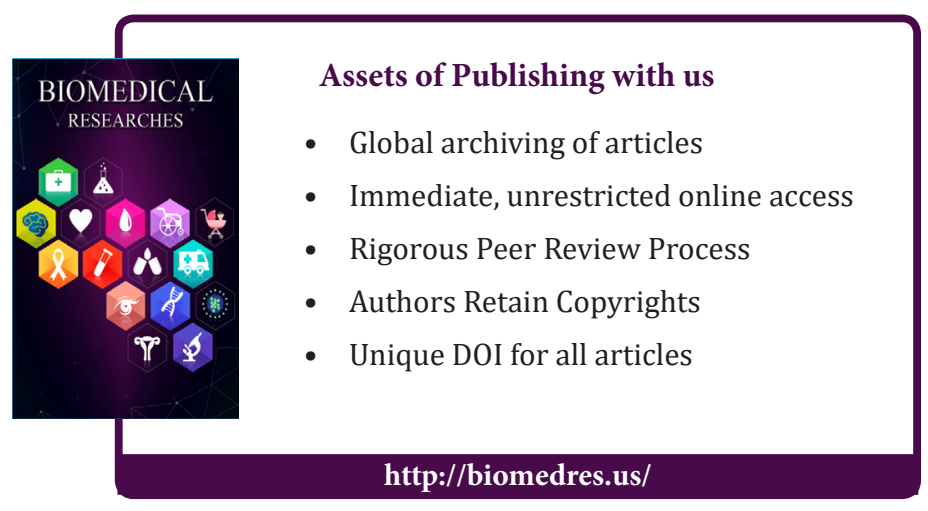

